EPHOU-93004

December 1993

\title{
2D Quantum Gravity in the Proper-Time Gauge
}

\author{
Ryuichi NAKAYAMA * \\ Department of Physics, Faculty of Science, \\ Hokkaido University, Sapporo 060, Japan
}

\begin{abstract}
A two-loop (cylinder) amplitude of the $2 \mathrm{~d}$ pure gravity theory is obtained in the proper-time gauge $\left(g_{00}=1, g_{01}=g_{10}=0\right)$ in the continuum formulation. The constraint $T_{01}=0$ is solved and used to reduce the problem of field theory to that of quantum mechanics. This reduction can also be proved by using a conformal Ward identity. The amplitude depends on the lengths $l_{1}, l_{2}$ of the boundaries, the proper time $T$ and a non-negative integer $m$ associated with winding modes around the boundaries.
\end{abstract}

*nakayama@particle.phys.hokudai.ac.jp 
Much progress in the formulation of $2 \mathrm{~d}$ quantum gravity has been made since the equivalence of $2 \mathrm{~d}$ quantum gravity and dynamical triangulation (DT) [1] was established. [2] - 10 As for the problem of understanding 2d gravity solely in the continuum theory, there still remain several technical difficulties, although there are some important developments [1] - [15]. Especially, loop amplitudes have not been derived by the quantization of the gravitationally induced action in spite of some attempts 8 [16.

In this letter a two-loop (cylinder) amplitude of the pure gravity theory will be derived in the continuum formulation of $2 \mathrm{~d}$ gravity. For this purpose we should first specify a suitable gauge. A natural coordinate system on a cylinder is obtained by choosing its height as the direction of the time coordinate $x^{0}$ and the cycle perpendicular to it as that of the space coordinate $x^{1}$. In this coordinate frame we will make the following gauge fixing for the metric (proper-time gauge [17]):

$$
g_{00}=1, \quad g_{01}=g_{10}=0 .
$$

The component $g_{11}$ is denoted as $\gamma\left(x^{0}, x^{1}\right)$.

Let us first derive a conformal Ward identity. We start from the proper-time gauge (1) and perform an infinitesimal reparametrization of $x^{0}, \delta x^{0}=\epsilon\left(x^{0}\right)$. Then the metric is no longer of the form of (1). To return to (1) we should rescale the metric as $g_{\mu \nu} \rightarrow\left(1+2 \epsilon^{\prime}\right) g_{\mu \nu}$. The total change of $\sqrt{\gamma}$ is $\delta \sqrt{\gamma}=\epsilon^{\prime} \sqrt{\gamma}-\epsilon \partial_{0} \sqrt{\gamma}$. Since the action and the integration measure are invariant under reparametrizations but not under Weyl rescalings, we obtain the following Ward identity

$$
\begin{aligned}
& <\int d^{2} x \epsilon^{\prime}\left(x^{0}\right) \sqrt{g(x)}(c R(x)+\lambda) \sqrt{\gamma\left(x_{1}\right)} \cdots \sqrt{\gamma\left(x_{n}\right)}> \\
= & \sum_{j=1}^{n}\left[-\epsilon\left(x_{j}^{0}\right) \partial / \partial x_{j}^{0}+\epsilon^{\prime}\left(x_{j}^{0}\right)\right]<\sqrt{\gamma\left(x_{1}\right)} \cdots \sqrt{\gamma\left(x_{n}\right)}>,
\end{aligned}
$$

where $\sqrt{g} R=-2 \partial_{0}^{2} \sqrt{\gamma}, c$ is a constant proportional to the conformal anomaly and $\lambda$ a cosmological constant. By removing $\epsilon$ from (2), integrating over $x_{j}^{1}$ and defining a length of the loop at time $x^{0}$ by $l\left(x^{0}\right)=\int_{0}^{\pi} d x^{1} \sqrt{\gamma(x)}$, we have

$$
\begin{aligned}
& {\left[-2 c\left(\partial / \partial x^{0}\right)^{3}+\lambda \partial / \partial x^{0}\right]<l\left(x^{0}\right) l\left(x_{1}^{0}\right) \cdots l\left(x_{n}^{0}\right)>} \\
= & \sum_{j=1}^{n}\left[\delta\left(x^{0}-x_{j}^{0}\right) \partial / \partial x_{j}^{0}+\delta^{\prime}\left(x^{0}-x_{j}^{0}\right)\right]<l\left(x^{0}\right) l\left(x_{1}^{0}\right) \cdots l\left(x_{n}^{0}\right)>.
\end{aligned}
$$

This is a closed equation for Green functions of $l\left(x^{0}\right)$. Existence of such an identity suggests that there should be a quantum-mechanical action for $l\left(x^{0}\right)$. Indeed there is such an action and it can be written as

$$
S_{l}=\int d x^{0}\left[\frac{c}{l}\left(\frac{d}{d x^{0}} l\right)^{2}+\frac{\lambda}{2} l+\frac{a}{l}\right]
$$


where $a$ is some constant whose value is unknown at present. It is easy to derive (3) from the action (雨) by a change of variables $l\left(x^{0}\right) \rightarrow l\left(x^{0}\right)+\epsilon^{\prime}\left(x^{0}\right) l\left(x^{0}\right)-\epsilon\left(x^{0}\right) l^{\prime}\left(x^{0}\right)$. One of the purposes of this letter is to derive (四) from the gravitationally induced action. It will be shown that the constant $a$ takes discrete values.

As is well-known, the continuum action in $2 \mathrm{~d}$ gravity is given by the induced action which is obtained by integrating out the matter fields coupled to gravity. In the conformal gauge $g_{\mu \nu}=e^{\phi} \hat{g}_{\mu \nu}$, the induced action coincides with Liouville action

$$
S_{L}\left(\phi ; \hat{g}_{\mu \nu}\right)=\int d^{2} x \sqrt{\hat{g}}\left[\frac{1}{4} \hat{g}^{\mu \nu} \partial_{\mu} \phi \partial_{\nu} \phi+\frac{1}{2} \phi \hat{R}+4 \lambda e^{\phi}\right],
$$

where $\phi$ is the conformal mode, $\hat{g}_{\mu \nu}$ is the reference metric and $\lambda$ is a cosmological constant. Although $S_{L}$ is local, in gauges such as (1) the induced action $S_{\text {ind }}$ is nonlocal. Let us parametrize an arbitrary metric $g_{\mu \nu}$ as follows:

$$
g_{\mu \nu} d x^{\mu} d x^{\nu}=e^{\phi} \hat{g}_{\mu \nu} d x^{\mu} d x^{\nu}=e^{\phi}(d z+\mu d \bar{z})(d \bar{z}+\bar{\mu} d z),
$$

where $z=x^{0}+i x^{1}, \bar{z}=x^{0}-i x^{1}$. We introduce two functions $f\left(x^{0}, x^{1}\right)$ and $\bar{f}\left(x^{0}, x^{1}\right)$ to define $\mu$ and $\bar{\mu}$ :

$$
\mu=\bar{\partial} f / \partial f \quad \text { and } \quad \bar{\mu}=\partial \bar{f} / \bar{\partial} \bar{f},
$$

where $\partial=\left(\partial_{0}-i \partial_{1}\right) / 2, \bar{\partial}=\left(\partial_{0}+i \partial_{1}\right) / 2$ and $\partial_{\mu}=\partial / \partial x^{\mu}$. The induced action $S_{\text {ind }}$ is then given 18 by

$$
S_{i n d}=S_{L}\left(\phi ; \hat{g}_{\mu \nu}\right)+S_{f}(f)+\bar{S}_{f}(\bar{f})+S_{l . c .}(\mu, \bar{\mu}),
$$

where $S_{f}(f)$ is Polyakov's light-cone gauge action [19]:

$$
S_{f}(f)=\frac{1}{2} \int d^{2} x\left[\frac{\partial^{2} f \partial \bar{\partial} f}{(\partial f)^{2}}-\frac{\partial^{3} f \bar{\partial} f}{(\partial f)^{2}}\right] .
$$

Here $\bar{S}_{f}(\bar{f})$ is the complex conjugate of $S_{f}(f)$. The local counterterms are determined by the requirement of general coordinate invariance and we have

$$
S_{l . c .}(\mu, \bar{\mu})=\int d^{2} x(1-\mu \bar{\mu})^{-1}\left\{2 \partial \mu \bar{\partial} \bar{\mu}-\mu(\bar{\partial} \bar{\mu})^{2}-\bar{\mu}(\partial \mu)^{2}\right\} .
$$

The action (\&) is indeed invariant under an infinitesimal coordinate transformation $(\delta z=\epsilon(z, \bar{z}))$ 円

$$
\begin{aligned}
\delta \phi & =\epsilon \partial \phi+(\partial+\bar{\mu} \bar{\partial}) \epsilon, \\
\delta \mu & =\epsilon \partial \mu+(\bar{\partial}-\mu \partial) \epsilon, \\
\delta \bar{\mu} & =\epsilon \partial \bar{\mu}+\left(\bar{\mu} \partial-\bar{\mu}^{2} \bar{\partial}\right) \epsilon, \\
\delta f & =\epsilon \partial f, \quad \delta \bar{f}=\epsilon \partial \bar{f} .
\end{aligned}
$$

\footnotetext{
${ }^{1}$ Here $\delta \bar{z}=\bar{\epsilon}(z, \bar{z})$, which is a complex conjugate of $\epsilon(z, \bar{z})$, is formally set to zero. It is a straightforward task to recover $\bar{\epsilon}$.
} 
Because $f$ and $\bar{f}$ are not local functions of $\mu$ and $\bar{\mu}, S_{\text {ind }}$ is non-local.

We note that in the gauge (1) $\mu, \bar{\mu}$ and $\phi$ are expressed in terms of $\sqrt{\gamma}$ as

$$
\mu=\bar{\mu}=(1-\sqrt{\gamma}) /(1+\sqrt{\gamma}), \quad \phi=2 \ln \{(1+\sqrt{\gamma}) / 2\}
$$

Conversely, $\gamma$ is a function of $f$ and $\bar{f}$, and by using (7) we obtain

$$
\sqrt{\gamma}=-i \partial_{1} f / \partial_{0} f=i \partial_{1} \bar{f} / \partial_{0} \bar{f}
$$

Thus $f$ and $\bar{f}$ are not independent. In the case of pure gravity the action $S$ consists of the cosmological term and the induced action coming from the Faddeev-Popov determinant. Here the ghost coordinates are integrated out. By substituting (12) into (8), we obtain

$$
\begin{aligned}
S= & \frac{\kappa}{4 \pi} \int d^{2} x\left[(3+3 \sqrt{\gamma}+\gamma)(1+\sqrt{\gamma})^{-3}\left(\partial_{0} \sqrt{\gamma}\right)^{2}-(1+\sqrt{\gamma})^{-3}\left(\partial_{1} \sqrt{\gamma}\right)^{2}\right. \\
& \left.+\frac{1}{2}\left\{\frac{\partial^{2} f \partial \bar{\partial} f-\partial^{3} f \bar{\partial} f}{(\partial f)^{2}}+\text { c.c. }\right\}+4 \lambda \sqrt{\gamma}\right]
\end{aligned}
$$

where $S_{\text {ind }}$ is multiplied by some constant, $\kappa /(4 \pi)$, which is proportional to the conformal anomaly.

We would like to reduce the problem of two-dimensional field theory to that of quantum mechanics. This will be done by using the fact that the stress-energy tensor must vanish. We will first modify the gauge condition of (1) to

$$
g_{00}=1+h_{00}, \quad g_{01}=h_{01},
$$

where $h_{00}$ and $h_{01}$ are some fixed functions. The partition function $Z$, which is reparametrization invariant, should be independent of $h_{00}$ and $h_{01}$ and we have the condition [12]

$$
\left.\frac{\delta Z}{\delta h_{00}(x)}\right|_{h_{00}=h_{01}=0}=\left.\frac{\delta Z}{\delta h_{01}(x)}\right|_{h_{00}=h_{01}=0}=0 .
$$

This is equivalent to the vanishing of the stress-energy tensor $T_{00}=T_{01}=0$. By differentiating $Z$ w.r.t. $h_{00}$ and $h_{01}$ arbitrary times we conclude that correlation functions of arbitrary number of $T_{00}$ 's and $T_{01}$ 's are zero. of Therefore we can substitute the solution of $T_{00}=0$ and/or $T_{01}=0$ into the action (14). In this letter we will solve only the constraint $T_{01}=0$ P explicitly and use this constraint to reduce the model (14) to quantum mechanics. Validity of this procedure will be justified later by the result (34), which coincides with (4).

\footnotetext{
${ }^{2}$ Contact terms may appear because in the action (8) the time components $g^{00}$ and $g^{01}$ are not simply the Lagrange multipliers of the constraints $T_{00}=T_{01}=0$. We will neglect such contact terms.

${ }^{3}$ It is known that the constraint $T_{00}=0$ is not simply satisfied.
} 
The stress-energy tensor of the model (14) is obtained by varying (8) w.r.t. $g^{\mu \nu}$ and we have

$$
\begin{aligned}
\frac{4 \pi}{\kappa} T_{00} & =\frac{1}{2}(F+\bar{F})+\frac{1}{\sqrt{\gamma}} \partial_{0}^{2} \sqrt{\gamma}-2 \lambda, \\
\frac{4 \pi}{\kappa} T_{01} & =\frac{i}{2} \sqrt{\gamma}(F-\bar{F}), \\
\frac{4 \pi}{\kappa} T_{11} & =-\frac{1}{2} \gamma(F+\bar{F})-2 \lambda \gamma,
\end{aligned}
$$

where

$$
F=\left\{f, x^{0}\right\}=\partial_{0}^{3} f / \partial_{0} f-\frac{3}{2}\left(\partial_{0}^{2} f / \partial_{0} f\right)^{2}, \quad \bar{F}=\left\{\bar{f}, x^{0}\right\}
$$

are Schwarzian derivatives.

The solution to the constraint $T_{01}=0$ turns out to be

$$
\begin{aligned}
& f\left(x^{0}, x^{1}\right)=A \tanh \left[\int_{0}^{x^{0}} \frac{d s}{\sqrt{\gamma_{0}(s)}}+i \int_{0}^{x^{1}} d \sigma \sqrt{\gamma_{1}(\sigma)}\right]+C, \\
& \gamma\left(x^{0}, x^{1}\right)=\gamma_{0}\left(x^{0}\right) \gamma_{1}\left(x^{1}\right),
\end{aligned}
$$

where $\gamma_{0}\left(x^{0}\right)$ and $\gamma_{1}\left(x^{1}\right)$ are arbitrary functions of $x^{0}$ and $x^{1}$, respectively, and $A$ and $C$ are arbitrary constants. This will be proved below. Before that, two remarks are in order. First, $\gamma$ is determined from (21) by using (13) and there is no ambiguity associated with multiplying $\gamma_{0}$ by a constant and dividing $\gamma_{1}$ by the same constant. Secondly, $f$ should be a single-valued function on a cylinder. Thus from (21) we conclude that $\gamma_{1}$ has to satisfy a condition

$$
\int_{0}^{\pi} d x^{1} \sqrt{\gamma_{1}\left(x^{1}\right)}=(m+1) \pi \quad(m=0,1,2, \ldots),
$$

where $0 \leq x^{1} \leq \pi$ is a cycle of the cylinder. Although we can transform $\gamma_{1}\left(x^{1}\right)$ into a constant $(m+1)^{2}$ by reparametrizations of $x^{1}$, two $\gamma_{1}$ 's corresponding to different values of $m$ cannot be connected by reparametrizations. Thus in order to compute a cylinder amplitude, we have to do summation over $m$.

Let us now prove (21-22). By using (13) we can show the following identities by direct calculations

$$
\begin{aligned}
& \partial_{1} F-i \sqrt{\gamma} \partial_{0} F-2 i \partial_{0} \sqrt{\gamma} F=i \partial_{0}^{3} \sqrt{\gamma} \\
& \partial_{1} \bar{F}+i \sqrt{\gamma} \partial_{0} \bar{F}+2 i \partial_{0} \sqrt{\gamma} \bar{F}=-i \partial_{0}^{3} \sqrt{\gamma}
\end{aligned}
$$

Because of (18) the constraint $T_{01}=0$ means that $F=\bar{F}$, and hence we can show from $(24)+(25)$ that $F$ is a function of only $x^{0}$. Then (24) can be integrated w.r.t. $x^{0}$

$$
F\left(x^{0}\right)=\bar{F}\left(x^{0}\right)=-\frac{1}{\sqrt{\gamma}} \partial_{0}^{2} \sqrt{\gamma}+\frac{1}{2 \gamma}\left(\partial_{0} \sqrt{\gamma}\right)^{2}-\frac{2}{\gamma} \gamma_{1}\left(x^{1}\right)
$$


where $\gamma_{1}\left(x^{1}\right)$ is an arbitrary real function of $x^{1}$. The non-linear differential equation for $f$ obtained by combining (20) and (26) has a general solution of the form

$$
f\left(x^{0}, x^{1}\right)=A\left(x^{1}\right) \tanh \left[\sqrt{\gamma_{1}\left(x^{1}\right)} \int_{0}^{x^{0}} \frac{d s}{\sqrt{\gamma\left(s, x^{1}\right)}}+B\left(x^{1}\right)\right]+C\left(x^{1}\right),
$$

where $A\left(x^{1}\right), B\left(x^{1}\right)$ and $C\left(x^{1}\right)$ are arbitrary functions of $x^{1}$. Finally by requiring that (27) should satisfy (13), we obtain (22) and find that $A$ and $C$ are constants and that

$$
B\left(x^{1}\right)=i \int_{0}^{x^{1}} d \sigma \sqrt{\gamma_{1}(\sigma)}
$$

We will next reduce the model (14) to quantum mechanics by using the solution (21-23) to the constraint $T_{01}=0$. . The simplest way to do this is to compute the Hamiltonian

$$
H_{\text {red }}=\int_{0}^{\pi} d x^{1} 2 \sqrt{\gamma} T_{00} .
$$

By using (17), (22), (23) and (26), we obtain

$$
H_{\text {red }}=\frac{\kappa}{2}(m+1)\left[\frac{1}{2 \sqrt{\gamma_{0}}}\left(\frac{d}{d x^{0}} \sqrt{\gamma_{0}}\right)^{2}-2 \lambda \sqrt{\gamma_{0}}-\frac{2}{\sqrt{\gamma_{0}}}\right] .
$$

Then the action which yields $H_{\text {red }}$ is found to be

$$
S_{\text {red }}=\frac{\kappa}{2}(m+1) \int_{0}^{T} d x^{0}\left[\frac{1}{2 \sqrt{\gamma_{0}}}\left(\frac{d}{d x^{0}} \sqrt{\gamma_{0}}\right)^{2}+2 \lambda \sqrt{\gamma_{0}}+\frac{2}{\sqrt{\gamma_{0}}}\right],
$$

where $T$ is the proper time. f The variation of $S_{r e d}$ w.r.t. $\gamma_{0}$ satisfies the following relation:

$$
\delta S_{\text {red }}=\int d^{2} x \sqrt{\gamma} \tilde{T}_{11} \delta\left(\frac{1}{\gamma}\right)
$$

where $\tilde{T}_{11}$ is obtained by substituting (26) into (19). Let us define the length of a loop at time $x^{0}$ by

$$
l\left(x^{0}\right)=\frac{1}{\pi} \int_{0}^{\pi} d x^{1} \sqrt{\gamma(x)}=(m+1) \sqrt{\gamma_{0}\left(x^{0}\right)} .
$$

Then the action (31) is rewritten in terms of $l$ as

$$
S_{\text {red }}=\kappa \int_{0}^{T} d x^{0}\left[\frac{1}{4} l^{-1}\left(x^{0}\right)\left\{\frac{d}{d x^{0}} l\left(x^{0}\right)\right\}^{2}+\lambda l\left(x^{0}\right)+(m+1)^{2} l^{-1}\left(x^{0}\right)\right] .
$$

\footnotetext{
${ }^{4}$ This procedure is subtle because $T_{01}$ and $T_{00}$ do not commute. Direct substitution of (21-23) into (14) does not yield the last term in (31). This term, however, should exist because it is identified as the Casimir energy for the cylindrical configuration.

${ }^{5}$ It was suggested in [8] without proof that the problem of 2 d gravity reduces to that of quantum mechanics. Classically $S_{\text {red }}$ is related to the minisuperspace model [8] in the conformal gauge by a transformation $x^{0} \rightarrow \tilde{x}^{0}$ with $d x^{0} / d \tilde{x}^{0}=\sqrt{\gamma_{0}}=\exp (\phi / 2)$.
} 
Note that $S_{\text {red }}$ coincides with (4) up to constant rescalings of the variables and that $a$ takes descrete values $\kappa(m+1)^{2}$.

In the conformal gauge the constant $\kappa$ is determined by invariance of the partition function under the transformation $\phi(x) \rightarrow \phi(x)+\epsilon(x), \hat{g}_{\mu \nu}(x) \rightarrow e^{-\epsilon(x)} \hat{g}_{\mu \nu}(x)$ [11]. In the light-cone gauge the SL(2) current algebra determines $\kappa$ [12]. In the present case an argument which determines the value of $\kappa$ is not available. Here we will set $\kappa=1$, because this leads to a simple and interesting two-loop amplitude. It is straightforward to consider the case of arbitrary $\kappa$. Later we will also consider the case $\kappa=\infty$. We also have to take into account the possibility that the coefficient $m+1$ in (31) may be additively renormalized by a Jacobian associated with a change of variables from $f\left(x^{0}, x^{1}\right)$ to $\gamma_{0}\left(x^{0}\right)$. It turns out that the action (34) with $m+1$ shifted by $-\frac{1}{2}$,

$$
S_{m}=\kappa \int_{0}^{T} d x^{0}\left[\frac{1}{4 l\left(x^{0}\right)}\left(\frac{d}{d x^{0}} l\left(x^{0}\right)\right)^{2}+\lambda l\left(x^{0}\right)+\frac{\left(m+\frac{1}{2}\right)^{2}}{l\left(x^{0}\right)}\right] \quad(\kappa=1)
$$

leads to the two-loop amplitude derived in matrix models.

To quantize the model (35) we will switch to the Minkowskian metric temporarily. The Hamiltonian $H_{m}$ derived from $S_{m}$ is given by

$$
H_{m}=\Pi_{l} l \Pi_{l}+\left(m+\frac{1}{2}\right)^{2} l^{-1}+\lambda l,
$$

where $\Pi_{l}$ is the canonical momentum conjugate to $l$ and is replaced by $-i \partial / \partial l$ upon quantization. We would like to solve the problem of energy eigenvalues

$$
H_{m} \Psi(l)=E \Psi(l) .
$$

Rescaling the variable $l \rightarrow z=2 \sqrt{\lambda} l$ and defining a new function $\Phi(z)=e^{z / 2} z^{-\left(m+\frac{1}{2}\right)} \Psi(l)$, we can rewrite (37) into a confluent hypergeometric differential equation

$$
z \frac{d^{2}}{d z^{2}} \Phi+(2 m+2-z) \frac{d}{d z} \Phi-\left(m+1-\frac{E}{2 \sqrt{\lambda}}\right) \Phi=0 .
$$

The wave function should be regular at both $l=0$ and $l=+\infty$. Solving (38) with this boundary condition, we obtain the following orthonormal wave functions and energy eigenvalues

$$
\begin{aligned}
\Psi_{n}^{(m)}(l) & =\sqrt{\frac{n !}{(n+2 m+1) !}}(2 \sqrt{\lambda})^{m+1} e^{-\sqrt{\lambda} l} l^{m+\frac{1}{2}} L_{n}^{(2 m+1)}(2 \sqrt{\lambda} l), \\
E_{n}^{(m)} & =2 \sqrt{\lambda}(n+m+1),
\end{aligned}
$$

where $n=0,1,2, \ldots$ and $L_{n}^{(\alpha)}(z)$ is Laguerre polynomial [20]. 
We now turn to the construction of the cylinder amplitude

$$
A_{m}\left(l_{1}, l_{2} ; T\right)=<l_{2}\left|e^{-T H_{m}}\right| l_{1}>=\sum_{n=0}^{\infty} \Psi_{n}^{(m)}\left(l_{2}\right) e^{-E_{n}^{(m)} T} \Psi_{n}^{(m)}\left(l_{1}\right) .
$$

As mentioned above, however, we have to do summation over $m$ in order to take all inequivalent classes of $\gamma_{1}\left(x^{1}\right)$ into account. The full amplitude will be given by

$$
A\left(l_{1}, l_{2} ; T\right)=\sum_{m=0}^{\infty} w_{m} A_{m}\left(l_{1}, l_{2} ; T\right),
$$

where $w_{m}$ is a weight factor. Let us first compute $A_{m}\left(l_{1}, l_{2} ; T\right)$. By using (3941) and a formula [20]

$$
\begin{aligned}
& \sum_{n=0}^{\infty} \frac{n !}{\Gamma(n+\alpha+1)} L_{n}^{(\alpha)}(x) L_{n}^{(\alpha)}(y) z^{n} \\
& =(1-z)^{-1} \exp \left\{-z(1-z)^{-1}(x+y)\right\}(x y z)^{-\alpha / 2} I_{\alpha}\left(2(1-z)^{-1} \sqrt{x y z}\right),|z|<1(
\end{aligned}
$$

we obtain

$$
\begin{aligned}
A_{m}\left(l_{1}, l_{2} ; T\right)= & \sqrt{\lambda} \operatorname{cosech}(\sqrt{\lambda} T) \exp \left[-\sqrt{\lambda}\left(l_{1}+l_{2}\right) \operatorname{coth}(\sqrt{\lambda} T)\right] \\
& \cdot I_{2 m+1}\left(2 \sqrt{\lambda} \sqrt{l_{1} l_{2}} \operatorname{cosech}\{\sqrt{\lambda} T\}\right),
\end{aligned}
$$

where $I_{\nu}(z)$ and $K_{\nu}(z)$ in (45) below are modified Bessel functions. To compare our result with that of matrix models, (44) should be integrated over $T$ from 0 to $+\infty$. By using a formula in 21] we have

$$
G_{m}\left(l_{1}, l_{2}\right)=\int_{0}^{\infty} d T A_{m}\left(l_{1}, l_{2} ; T\right)=K_{m+\frac{1}{2}}\left(\sqrt{\lambda} l_{a}\right) I_{m+\frac{1}{2}}\left(\sqrt{\lambda} l_{b}\right),
$$

where $a=1, b=2$ if $l_{1}>l_{2}$ and $a=2, b=1$ otherwise. This result was in fact expected, because $G_{m}\left(l_{1}, l_{2}\right)$ is the solution of

$$
H_{m}\left(l_{1}, \partial_{l_{1}}\right) G_{m}\left(l_{1}, l_{2}\right)=\delta\left(l_{1}-l_{2}\right) .
$$

Let us now consider the full amplitude (42). We do not have a definite argument to determine $w_{m}$. If we set for instance

$$
w_{m}=(-1)^{m}(2 m+1),
$$

then by (42) and (45) we obtain

$$
\begin{aligned}
\int_{0}^{\infty} d T A\left(l_{1}, l_{2} ; T\right) & =\sum_{m=0}^{\infty}(-1)^{m}(2 m+1) \int_{0}^{\infty} d T A_{m}\left(l_{1}, l_{2} ; T\right) \\
& =\sum_{m=0}^{\infty}(-1)^{m}(2 m+1) K_{m+\frac{1}{2}}\left(\sqrt{\lambda} l_{a}\right) I_{m+\frac{1}{2}}\left(\sqrt{\lambda} l_{b}\right) .
\end{aligned}
$$


Due to the following Gegenbauer's addition formula [20] [8],

$$
\begin{aligned}
K_{\nu}(R) R^{-\nu} & =2^{\nu} \Gamma(\nu) \sum_{n=0}^{\infty}(\nu+n) C_{n}^{1 / 2}(\cos \theta) K_{\nu+n}(z) I_{\nu+n}(\zeta)(z \zeta)^{-\nu} \\
R & =\left(z^{2}+\zeta^{2}-2 z \zeta \cos \theta\right)^{1 / 2}, \quad|\zeta|<|z|, \quad \nu \neq 0,-1,-2, \ldots
\end{aligned}
$$

(48) can be summed to yield

$$
\int_{0}^{\infty} d T A\left(l_{1}, l_{2} ; T\right)=\frac{\sqrt{l_{1} l_{2}}}{l_{1}+l_{2}} e^{-\sqrt{\lambda}\left(l_{1}+l_{2}\right)},
$$

where $C_{n}^{\nu}(z)$ is Gegenbauer polynomial. This agrees with the result of matrix model calculations [8]. However, the two-loop amplitude derived in matrix models contains diagrams with many branches, i.e. small universes, attached on the wall of the cylinder. In fact the analysis of [9] shows that space-time geometries with infinitely many, very thin branches prevail in the cylinder amplitude. On such geometries the gauge (11) cannot be chosen and hence the calculations in the present letter and those in matrix models cannot be compared. Therefore we have to choose different $w_{m}$ and determination of the value of $w_{m}$ is left to the future work.

Up to this point we have assumed that $\kappa=1$. However the Faddeev-Popov determinant in the gauge (11) is given by

$$
\Delta_{F P}=\left[\operatorname{det}^{\prime}\left(-\gamma^{-\frac{1}{2}} \partial_{0} \gamma^{\frac{1}{2}} \partial_{0}\right) \operatorname{det}\left(-\gamma^{-\frac{3}{2}} \partial_{0} \gamma^{\frac{3}{2}} \partial_{0}\right)\right]^{\frac{1}{2}}
$$

where det $^{\prime}$ implies the determinant for nonzero modes. The operators in (51) do not contain $\partial_{1}$ and hence (51) is ill-defined. Therefore the value of $\kappa$ may be infinite. In such a case we have to keep $\kappa$ in the above arguments. The amplitude (44) now reads

$$
\begin{aligned}
A_{m}\left(l_{1}, l_{2} ; T\right)= & \kappa \sqrt{\lambda} \operatorname{cosech}(\sqrt{\lambda} T) \exp \left[-\kappa \sqrt{\lambda}\left(l_{1}+l_{2}\right) \operatorname{coth}(\sqrt{\lambda} T)\right] \\
& \cdot I_{\kappa(2 m+1)}\left(2 \kappa \sqrt{\lambda} \sqrt{l_{1} l_{2}} \operatorname{cosech}(\sqrt{\lambda} T)\right) .
\end{aligned}
$$

The $\kappa \rightarrow \infty$ limit of (52) depends on how $l_{1}, l_{2}$ and $\lambda$ are rescaled. If $l_{1}, l_{2}$ and $\lambda$ are kept fixed, then the $\kappa \rightarrow \infty$ limit of (52) is given by

$$
A_{m}\left(l_{1}, l_{2} ; T\right) \sim \sqrt{\frac{\kappa\left(m+\frac{1}{2}\right)}{2 \pi l_{1} l_{2} \sinh (2 \xi)}} \exp (-\kappa U),
$$

where

$$
U=\sqrt{\lambda}\left(l_{1}+l_{2}\right) \operatorname{coth}(\sqrt{\lambda} T)-2\left(m+\frac{1}{2}\right) \operatorname{coth} \xi+2\left(m+\frac{1}{2}\right) \xi
$$

and $\xi$ is defined by

$$
\sinh \xi=\left(m+\frac{1}{2}\right)\left(\lambda l_{1} l_{2}\right)^{-\frac{1}{2}} \sinh (\sqrt{\lambda} T)
$$


If we instead rescale $l_{1}, l_{2}$ and $1 / \lambda$ by an infinite amount, that is, if we replace $l_{j}$ and $1 / \lambda$ by $\Lambda l_{j}$ and $\Lambda^{\prime} / \lambda$, respectively, where $\Lambda$ and $\Lambda^{\prime}$ go to $\infty$ as $\kappa \rightarrow \infty$, then we have simply

$$
\lim _{\kappa \rightarrow \infty} A_{m} \propto \delta\left(l_{1}-l_{2}\right) .
$$

This means that the Hamiltonian for loop propagation is zero.

To recapitulate, in the pure gravity theory on a cylinder the quantum-mechanical action (35) for the length $l\left(x^{0}\right)$ of a loop was derived by starting from the gravitationally induced action in the proper-time gauge (11) and solving the gauge constraint $T_{01}=0$. Then the two-loop amplitude $A_{m}\left(l_{1}, l_{2} ; T\right)$ (44) was obtained by the quantization of this model.

An interesting point is that the summation over $m$ in (42) is a natural consequence of the requirement of integration over all inequivalent classes of $\gamma_{1}\left(x^{1}\right)$. These degrees of freedom $m=0,1,2, \ldots$ are the modes associated with the cycle of the cylinder. It will be important to further clarify the meaning of these winding modes. Let us also note that the amplitude (41) satisfies the composition law

$$
\int_{0}^{\infty} d l_{2} A_{m}\left(l_{1}, l_{2} ; T\right) A_{m}\left(l_{2}, l_{3} ; T^{\prime}\right)=A_{m}\left(l_{1}, l_{3} ; T+T^{\prime}\right),
$$

while the full amplitude (42) fails to satisfy (57) due to the sum over $m$.

In this letter only the cylinder, i.e., the propagator was studied. As a next step, we would like to introduce the time $T$ and the quantum number $m$ to each boundary of a disk and a three-loop vertex. If this is achieved, higher genus amplitudes may be obtained by sewing the boundaries of vertices.

The author thanks N. Ishibashi, H. Kawai, Y. Okamoto and K. Yoshida for discussions. 


\section{References}

[1] V.A. Kazakov, I.K. Kostov and A.A. Migdal, Phys. Lett. 66 (1991) 2051;

J. Ambjørn, B. Durhuus and J. Fröhlich, Nucl. Phys. B257 [FS14] (1985) 433;

F. David, Nucl. Phys. B257 [FS14] (1985) 543.

[2] E. Brézin and V. Kazakov, Phys. Lett. B236 (1990) 144;

M. Douglas and S. Shenker, Nucl. Phys. B335 (1990) 635;

D. J. Gross and A. Migdal, Phys. Rev. Lett. 64 (1990) 127.

[3] M. Douglas, Phys. Lett. B238 (1990) 176.

[4] M. Fukuma, H. Kawai and R. Nakayama, Int. J. Mod. Phys. A6 (1991) 1385;

Commun. Math. Phys. 143 (1992) 371; Commun. Math. Phys. 148 (1992) 101.

[5] R. Dijkgraaf, E. Verlinde and H. Verlinde, Nucl. Phys. B348 (1991) 435.

[6] A. Jevicki and T. Yoneya, Mod. Phys. Lett. A5 (1990) 1615;

P. Ginsparg, M. Goulian, M. Plesser and J. Zinn-Justin, Nucl. Phys. B342 (1990) 539.

[7] T. Yoneya, Commun. Math. Phys. 144 (1992) 623; Int. J. Mod. Phys. A7 (1992) 4015;

J. Goeree, Nucl. Phys. B358 (1991) 737.

[8] G. Moore, N. Seiberg and M. Staudacher, Nucl. Phys. B362 (1991) 665.

[9] H. Kawai, N, Kawamoto, T. Mogami and Y. Watabiki, Phys. Lett. 306B (1993) 19.

[10] N. Ishibashi and H. Kawai, Phys. Lett. B314 (1993) 190.

[11] J. Distler and H. Kawai, Nucl. Phys. B321 (1989) 509;

F. David, Mod. Phys. Lett. A3 (1988) 1651.

[12] V.G. Knizhnik, A.M. Polyakov and A.B. Zamolodchikov, Mod. Phys. Lett. A3 (1988) 819.

[13] M. Goulian and M. Li, Phys. Rev. Lett. 66 (1991) 2051;

Y. Kitazawa, Phys. Lett. 265B (1991) 262;

N. Sakai and Y. Tanii, Prog. Theor. Phys. 86 (1991) 547;

A. Gupta, S. Trivedi and M. Wise, Nucl. Phys. B340 (1990) 475. 
[14] T. L. Curtright and C. B. Thorn, Phys. Rev. Lett. 48 (1982) 1309;

E. Braaten, T. Curtright and C. Thorn, Phys. Lett. 118B (1982) 115; Ann. Phys. 147 (1983) 365.

[15] J. -L. Gervais and A. Neveu, Nucl. Phys. B199 (1982) 59; B209 (1982) 125; B224 (1983) 329; B238 (1984) 396;

J. -L. Gervais, Phys. Lett. 243B (1990) 85; Commun. Math. Phys. 130 (1990) 257.

[16] N. Seiberg, Prog. Theor. Phys. Supple. 102 (1990) 319.

[17] C. Teitelboim, Phys. Rev. Lett. 50 (1983) 705;

T. Banks and L. Susskind, Int. J. of Theor. Phys. 23 (1984) 475.

[18] H. Verlinde, Nucl. Phys. B337 (1990) 652.

[19] A. M. Polyakov, Mod. Phys. Lett. A2 (1987) 893.

[20] A. Erdélyi, W. Magnus, F. Oberhettinger and F. G. Tricomi, Higher Transcendental Functions, Vol II, McGraw-Hill Book Co., 1953.

[21] I. Gradshtein and I. Ryzhik, Table of Integrals, Series and Products, Academic press, 1965 , formula 4 . of 6.669 . 